\title{
Dental considerations in Thalassemic patients
}

\author{
Dr Sakshi Madhok ${ }^{1}$, Dr Saksham Madhok ${ }^{2}$ \\ ${ }^{I}$ (Asstt Prof. Deptt of Prosthodontics, Z.A.D.C., A.M.U.) \\ ${ }^{2}$ (Post Graduate student, Deptt of Orthodontics, M.P.C.D.\& R.C., Gwalior, India)
}

\begin{abstract}
Thalassemia is one of the most confusing hemoglobinopathies. It is a kind of chronic inherited microcytic anemia characterized by defective hemoglobin synthesis and ineffective erythropoesis. It poses definite problems in relation to the dental treatment. Dental professionals should be aware of the nature and course of the disease and its implication on dental care and treatment. The severity of thalassemia varies from minimal anemia to transfusion dependence. Over the last twenty years, management for thalassemia major has improved to the point where we predict almost normal life expectancy of the patient, thus the provision of integral rather than palliative dental treatment must be considered. In this article orofacial, and non-skeletal manifestations of thalassemia are discussed with an overview of radiographic and dental considerations in such patients. The variable anemic condition of the patient, the transfusional and absorptive iron overload and the hyperplastic erythroid masses to compensate anemia complicate even the routine dental procedures in a thalassemic patient
\end{abstract}

Key Words: Anemia,,Chip-munk facies, Erythroid hyperplasia, Hair-on-end appearance, Rodent facies, Thalassemia.

\section{Introduction}

Thalassemia was described in 1927 by Cooley et al., is a kind of severe anemia associated with splenomegaly and bone abnormalities[1]. Homozygous beta thalassemia, also known as Cooley's anemia or Mediterranean anemia is chiefly seen in Mediterranean population[2]. World wide approximately 15 million people are expected to suffer from thalassemic disorders. In India nearly 12,000 infants are born every year with a hemoglobinopathy. On an average one in every 25 Indian is a carrier of thalassemia.

Lack of easy diagnostic tests, and its similarity to iron deficiency anemia makes thalassemia one of the most confusing hemoglobinopathy. Whereas both thalassemia and iron deficiency anemia are characterized by microcytic hypochromic anemia, remedy of iron deficiency anemia lies in iron supplementation, but iron supplementation does not correct the anemia due to thalassemia.

Thalassemia can be classified according to the chain involved. In beta thalassemia there is deficient synthesis of beta globin, whereas in alpha thalassemia there is deficient synthesis of alpha globin. Reduced synthesis of one of the two globin polypeptides leads to deficient hemoglobin accumulation, resulting in hypochromic and microcytic red cells.

Thalassemia trait, also known as thalassemia minor, is found in heterozygous individuals with impaired alpha and beta chain production. This does not generate clinical signs, and the presence of splenomegaly is rare.

In beta thalassemia, there is a large lack of normal beta chain production. This causes a relative excess of alpha chains, the latter are insoluble and tend to precipitate, forming intracellular inclusions that deform the structure of erythrocytes and lead to premature destruction within the bone marrow and spleen.

Beta thalassemia major, historically called Cooley's anemia, occurs when both genes necessary for beta globin production are affected. Beta thalassemia presents at six months of age when adult hemoglobin has replaced fetal hemoglobin. Peripheral anemia, caused by the disease, sends signals to the bone marrow to increase production of erythrocytes (via erythropoietin), however, erythrocyte production is abnormal. The process is called 'ineffective erythropoesis'. With time, the marrow cavities (skull bones, facial bones and ribs) expand (erythroid hyperplasia), leading to classical facial features and radiographic findings. Massive erythropoesis within the bones invades bony cortex, impairs bone growth and produces other skeletal abnormalities. Erythrocytes are noted to be abnormal by the reticulo-endothelial system, and are taken up by these organs resulting in enormous hepatoslenomegaly. In untreated patients, death usually occurs by the end of the second decade of life from anemia and congestive heart failure. These patients need regular transfusions to survive (every two to four weeks).

Thalassemia intermedia is the other form of severe beta thalassemia. These patients need blood transfusion but not regularly. The prognosis of such cases is much better than in patients with thalassemia major and dental treatment is comparatively less problematic. Diagnostic criteria for the major verses the intermedia syndrome are rather ill defined and are largely based on the hemoglobin level without transfusion[3]. 
Alpha-thalassemia results from deletion of any one of four genes necessary to make alpha globin chains. Since the non alpha chains in general form more soluble and less toxic aggregates, the hemolytic anemia and ineffective erythropoesis tend to be less severe than with beta thalassemia of similar degree of chain imbalance.

\section{Diagnosis Of Thalassemia}

Diagnosis of thalassemia involves a three tier approach[4].

i. Complete blood count - This initial haematological test gives a general idea of the cells in the blood stream. If Mean Corpuscular Volume and Mean Corpuscular Haemoglobin are low and iron deficiency has been ruled out, thalassemia should be considered. In thalassemia RBCs may show anisocytosis ,poikilocytosis, be nucleated and have uneven hemoglobin distribution (producing target cells that look like bull's eye under the microscope

ii. Thalassemia screen test or haemoglobinopathy test. This test measures the type and relative amounts of haemoglobin $(\mathrm{Hb})$ present in the RBCs. This test is done once a haemoglobinopathy is suspected based on family history or full blood count. $\mathrm{Hb} \mathrm{A}\left(\alpha_{2} \beta_{2}\right)$ is the normal type of $\mathrm{Hb}$ found in adults. A raised $\mathrm{Hb}$ $\mathrm{A}_{2}\left(\alpha_{2} \delta_{2}\right)$ is the key parameter indicating the presence of beta thalassemia. $\mathrm{Hb} \mathrm{F}\left(\alpha_{2} \gamma_{2}\right)$ is also raised in beta thalassemia. $\mathrm{Hb} \mathrm{H}$ inclusions ( $\beta_{4}$ tetramer) is a definitive test to confirm alpha thalassemia in adults. Bart haemoglobin ( $\gamma_{4}$ tetramer) is seen in new borns with $\alpha$ thalassemia.

iii. DNA mutation analysis- This test is used to investigate deletions and mutations in alpha and beta globin producing genes. This is not routinely used but is used when a haemoglobinopathy cannot be confirmed by the thalassemia screening test or when the underlying mutation is being looked for in a confirmed haemoglobinopathy.

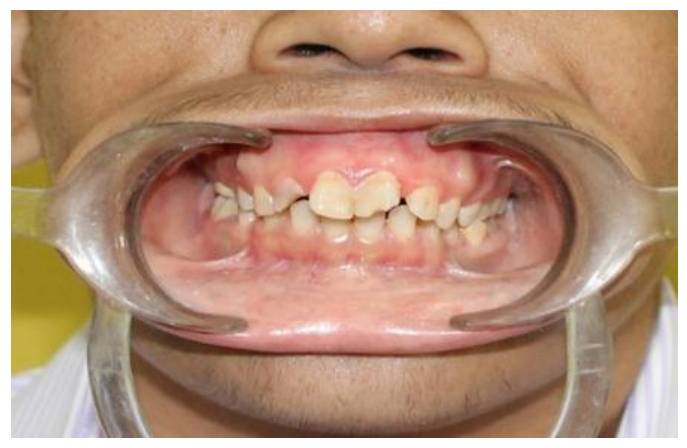

Figure1. Malocclusion in thalassemic patients

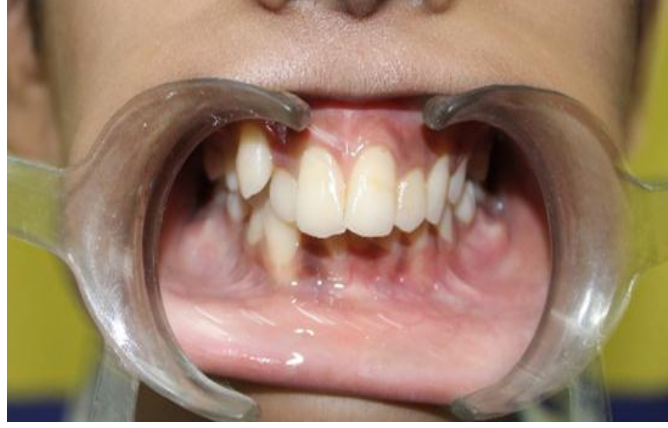

Figure 2. Malocclusion in thalassemic patients

\section{Orofacial Manifestations}

The most common orofacial manifestations are due to intense compensatory hyperplasia of the marrow and expansion of the marrow cavity[5]. Thalassemia major patients develop skeletal class II maloclussion subsequent to maxillary protrusion and mandibular atrophy. (Fig1,2) The early fusion of occipital sutures takes place concomitantly with medullary hyperplasia of the anterior maxillofacial structures, causing maxillary skeletal protrusion. Often the mandibular arch is telescoped within the maxillary arch (Brodie syndrome) in thalassemia major patients[6]. Malocclusion due to maxillary protrusion, increased overjet and anterior open bite, malar prominence, saddle nose and frontal bossing give an appearance of 'chip-munk facies' or rodent facies'[7]. (Fig3,4) The mandible is generally less protruded than maxilla apparently because the dense mandibular cortical layer resists expansion[8]. Overgrowth of marrow in frontal, temporal and facial bones consistently impedes pneumatisation of paranasal sinuses[9]. Marrow overgrowth in maxillary bone may cause lateral displacement of orbits (hyperteleorism)[9].

Other oral features include the appearance of spiky-shaped and short roots, taurodontism, multiple diastemas, splayed incisors, attenuated lamina dura, absence of inferior alveolar canal and thin cortex of the mandible.

Caries index is high in thalassemics not only because of oral hygiene neglect but also because median saliva concentrations of phosphorous and Ig A are significantly lower in such patients.

Mucosal pallor and atrophic glossitis is a constant finding especially when haemoglobin drops below $8 \mathrm{mg} / \mathrm{dl}$. Severe gingivitis is evident if the patient is subjected to splenectomy[10]. Iron deposits can cause painful inflammation of salivary glands with diminished salivary flow[11]. The colour of gingiva sometimes tends to be dark due to high ferritin levels in the blood. Macroglossia may be present due to bigger arches.

If blood transfusions have been carried out since birth, upto $50 \%$ of patients may either not present these manifestations or do so in a mild form[11]. 


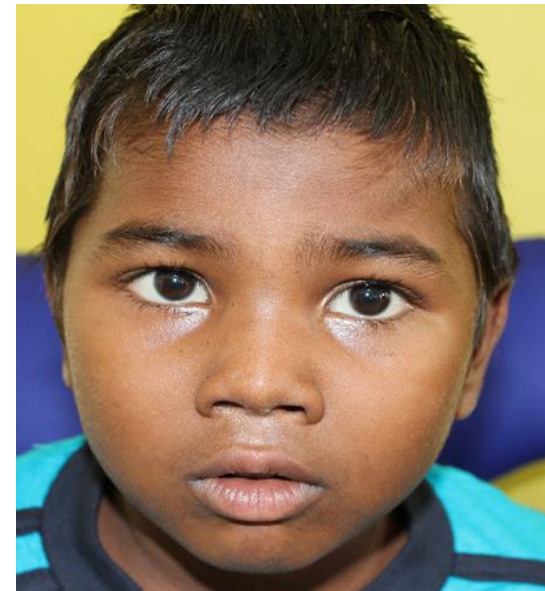

Figure 3. Chip-munk Facies

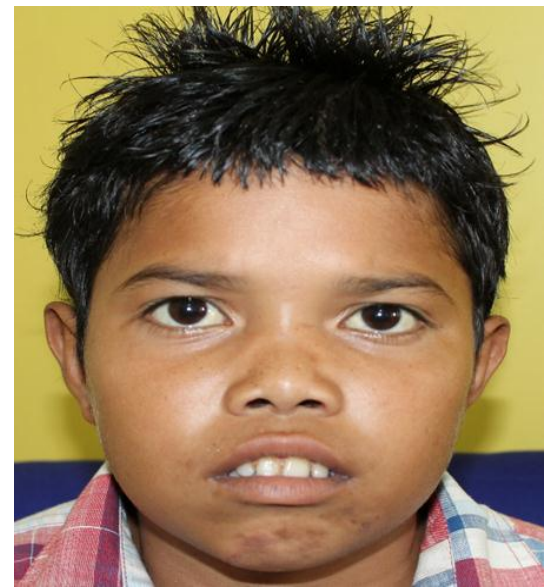

Figure 4. Chip- munk Facies.

III. Roentgenographic Features

These are also the result of marrow hyperplasia in response to chronic anemia. The skeletal response to this marrow proliferation consists of expansion of the medulla, thinning of the cortical bone and resorption of the cancellous bone resulting in generalized loss of density. The tubercle of the jaw bone seen in panoramic radiograph gives a honeycomb appearance[12]

Skull changes consists of widening of diploic spaces and displacement and thinning of the outer table. Sometimes the diploic trabeculae orient themselves perpendicular to the tables producing a radial pattern called hair-on-end appearance[13].

The frontal bones reveal the earliest and the most severe changes, where as the inferior bones usually remain unaltered. Hypertrophic marrow perforates cortex and extends longitudinally beneath the periosteum cloaking the original developing rib resulting in rib-within- rib appearance[10]. Premature fusion of the growth plates in the tubular bones of the extremities most commonly in the proximal humerus and distal femur represents a very common finding in children with thalassemia major[14].

\section{Treatment Of Thalassemia}

Currently, part of the standard treatment for beta thalassemia major is lifelong transfusions given every two to four weeks. The intent of these transfusions is to keep their hemoglobin above $9-10 \mathrm{gm} / \mathrm{dl}$. This will in effect shut off the patient's own ineffective erythropoesis. In general patients are loaded with iron at an average rate of $.5 \mathrm{mg} / \mathrm{kg}$ bw/day[15]. Iron (the one way element) in addition to being relatively difficult to absorb, is also not easily excreted. Thus, such transfused patients quickly become iron loaded. Untreated iron overload can be fatal. The repeated transfusions gradually increase the total body iron load, resulting in transfusional hemosiderosis with complications in the heart, endocrine glands and the liver. Infection with bacteria especially Yersinia and Klebsiella are more common in individuals who have excess body iron[16]. Thus a dentist must keep in mind the impending multiorgan complications and increased susceptibility to infection in a merely anemic appearing thalassemic patient.

\subsection{Assessment of iron overload:}

Methods are being developed to assess 'free' iron, the iron that is not bound to transferrin in the circulation or is free within cells and not attached to ferritin. This iron can cause tissue damage and may be detected by specific assays[17].

Ferritin is a metalloprotein found in cells. Normal values of serum ferritin are 12-300ng/ml. Thalassemic patients with serum ferritin values that are consistently less than $1000 \mathrm{ng} / \mathrm{ml}$ have a better prognosis[18].

Labile plasma iron(LPI) is a more reliable marker of the body iron status than ferritin. When the transferrin saturation is over 70\%-80\% labile plasma iron appears[19]. The LPI represents a component of non transferrin binding iron that is both redox active and chelatable. It permeates organs in due course and compromises organ function.

In transfusion dependent patients, the iron in the liver represents the total body iron load, as it stores $70 \%-80 \%$ of the iron[20]. Patients with high levels of Liver iron concentration (LIC) are at increased risk of developing cardiac iron compared with patients with low LIC[21].

Thus a thalassemic even with regular blood transfusions is not free of problems. The increasing iron stores with every transfusion is a threat to the functioning of patient's vital organs. A dentist should be vigilant 
in regards to the iron overload while treating a thalassemic patient, considering whether the patient is mild, moderately or heavily overloaded. Greater the iron load poorer the prognosis.

\subsection{Iron chelator:}

Regularly transfused patients need to be on life long chelation therapy to help their bodies excrete the excess iron. With the combination of transfusion and chelation therapy, life expectancy can be normal. Currently three iron chelators are available for use either as monotherapy or combination.

- Deferoxamine- Iron chelation was introduced with parentral deferoxamine. It binds iron in 1:1 ratio. As it is not orally absorbed and has a very short half life, the most common form of administration is by slow subcutaneous infusion by small portable pumps[22]. Dosage is $20-60 \mathrm{mg} / \mathrm{kg}$ bw/infusion $5-7$ days a week depending on the degree of iron load[23].

- Deferiprone- Introduced in 1999,orallyadministered deferiprone binds iron in the ratio of 3:1. Dose approved is $75-99 \mathrm{mg} / \mathrm{kgbw} / 24 \mathrm{hrs}[16]$, usually 8 hours apart.It is particularly effective in removing iron from the heart[24].

- Deferasirox- This oral chelator introduced in 2006 binds to iron in 2:1 ratio. Due to the relatively long half life, it may be administered in once daily dose, ranging between $10-40 \mathrm{mg} / \mathrm{kg}$ bw[16].

\subsection{Splenectomy:}

The presence of hypersplenism intensifies the need for blood transfusion. This worsens the problems posed by iron accumulation. The presence of leucopenia and thrombocytopenia hastens the decision to remove spleen. However, the sequel to splenectomy is the risk of sudden sepsis caused by encapsulated microorganisms. Such patients frequently receive daily continued prophylaxis.

\subsection{Elevation of fetal haemoglobin levels:}

Administration of recombinant human erythropoietin and Hydroxyurea increases gamma-chain synthesis to some extent, with a consequent rise in fetal hemoglobin[25]. But, these measures cannot substitute the blood transfusions [26].

\subsection{Alternative therapy:}

The quest to combat this disease led to bone marrow transplantation and stem cell therapy, but these too with its own limitations like HLA histocompatibility. The potential gene therapy may be expected to allow complete curing of patients[27] in the future thereby greatly simplifying the dental management of these patients. Need of the hour is prevention of thalassemia by prenatal screening.

\section{Dental Treatment Of Thalassemic Patients}

When subjecting a patient with thalassemia to dental treatment attention should be focused on obtaining as much information as possible to confirm safe dental treatment and adequate distinction among thalassemic subjects. Before beginning the treatment the following should be ascertained:

- Type of Thalassemia

- Present hemoglobin level of the patient

- Degree of iron overload in the body

- Relative organ involvement with iron

- Chelators administered to the patient

- Presence or absence of splenomegaly

- History of splenectomy

- Overall patient prognosis and life expectancy.

The treatment plan differs according to whether patient has thalassemia minor, major or intermedia. In patients with thalassemia trait more complex procedures can be considered. The diagnosis of thalassemia intermedia should not be mistakenly considered as benign by the dentist and proper coagulation and $\mathrm{Hb}$ level tests should be performed before undergoing any surgical procedure. In thalassemia major serial transfusions provide the back up securing coagulation and bleeding time with the fraction of transfused platelets. But this is not the case with intermedia.

Any invasive procedure in these patients should be done immediately after transfusion following mandatory antibiotic prophylaxis and the study of coagulation profile. Hb level should be more than $10 \mathrm{mg} \%$ before any clinical procedure. Complex surgical treatment are contraindicated in patients with thalassemia major if blood transfusion and chelator therapy has been shown to be less effective.

The common oral complaints of such patients are orofacial deformities and malocclusion. Such problems in patients with thalassemia intermedia and other less severe variants can be dealt with surgically 
including remodeling of the maxillary protuberance followed by orthodontic treatment for aligning the incisors. Maxillary osteotomy for repositioning of the maxilla can also be done for improved esthetic outcome.

As these patients commonly develop skeletal Class II malocclusion, it is recommended that orthodontic treatment be initiated as early as possible to lessen the effects of this syndrome. In thalassemics , preventive and interceptive orthodontics takes precedent over therapeutic orthodontics. Forces used in thalassemic patients should be low forces. Since the cortical plates are very thin, these patients should be followed more closely with shorter intervals between appointments. The retention phase is also difficult in such patients

Excess iron in the body increases the risk of fibrosis, cirrhosis and liver cancer[28]. The liver function and coagulation tests should be performed before dental procedures are carried out. This is to rule out any liver disorder that may have been caused by excess iron. Diminished coagulation factors could also be due to intense vitamin $\mathrm{K}$ absorption deficiencies due to prolonged antibiotic coverage or previously progressing hepatitis. Caution should be exercised by the dentist in employing the commonly administered hepatotoxic drugs. Tetracycline, metronidazole and erythromycin estolate should be avoided entirely. Paracetamol is a safe alternative to NSAIDS and aspirin.

The possibility of appearance of diabetes as an expression of secondary hemochromatosis requires strict preventive measures by the dentist which includes the assessment of sugar levels and antibiotic prophylaxis before undertaking any dental procedure. Periodontitis, gingivitis etc. are very dangerous foci of infection in these immune impaired individuals. This situation is worsened in the presence of splenectomy.

As mentioned splenectomy is one of the treatment modalities in a thalassemic patient. The presence or absence of this organ modifies the dental approach. In this context, the absence of hypersplenism, without leukopenia and thrombocytopenia, facilitates dental treatment. In contrast, the presence of hypersplenism with leucopenia and thrombocytopenia requires the provision of antibiotic and platelet concentrates before dental procedures.

In splenectomised individuals there is modest increase in circulating platelets and susceptibility to sepsis by bacteria and protozoa increases. The oral cavity must be prevented from being a source of bacterial spread, this requires increased radicalness in dental treatment and extreme caution in orthodontic practice. The increase in platelet count resulting from splenectomy implies a greater risk of thrombosis, the administration of anti platelet drugs in such cases requires monitorization of bleeding time or consultation with a hematologist. If the patient is taking penicillins regularly a change in antibiotic is recommended during dental treatment period, to avoid possible bacterial resistance.

Oral chelators are commonly used by thalassemic patients. The side effects of oral deferiprone includes hepatitis, agranulocytosis and neutropenia. Deferasirox affects primarily the kidney function and also causes hepatitis, gastrointestinal bleeding and cytopenia[16]. The possible involvement of immune system as side effect of these drugs gives one more reason why antibiotic coverage is mandatory in thalassemic patients. Extreme caution must be taken in the drug type and dosage such as non steroidal anti-inflammatory drugs and certain antibiotics as the liver and kidney functions are altered with the possible presence of gastrointestinal bleeding.

The complexity of these patients makes sedation/anaesthesia an important aspect when planning even the simplest of dental treatments, to ensure improved pre-,intra-,and post-operative control.

Dental management of a thalassemic patient requires special concern as this patient could be a heart patient, a diabetic, a splenectomised or a patient with compromised immunity and liver function. A dentist should have profound knowledge to manage this medically compromised state of a thalassemic patient. The best way is to have a multidisciplinary approach involving a dental surgeon, a haematologist and an orthodontist to safely provide dental treatment to these patients with seemingly benign blood dyscrasia.

\section{References}

[1]. Cooley TB, Witwer ER, Lee P. Anemia in children with splenomegaly and peculiar changes in the bones. Am J Dis Child $1927 ; 34: 347$

[2]. Weatherall JD, Clegg JB. The thalassaemia syndromes $\left(3^{\text {rd }}\right.$ ed, Oxford: Blackwell Scientific: 1981). p.132-74

[3]. Ronald J A Trent. Diagnosis of Hemoglobinopathies. Clin Biochem Rev 2006;27:27-38

[4]. Nienhuis AW, Nathan DG. Pathophysiology and clinical manifestations of the $\beta$ - Thalassemias. Available www.perspectiveinmedicine.org on July 3, 2013

[5]. Ronald J A Trent. Diagnosis of the haemoglobinopathies. Clin Biochem Rev 2006;27:27-38

[6]. Adeyemo TA, Adeyemo WL, Adediran A et.al. Orofacial manifestations of hematological disorders: Anemia and hemostatic disorders. Indian Journal of Dental Research 2011;22:454-461

[7]. Kharsa MA. Orthodontic Characteristics of Thalassemia Patients:Orthod Cyber Journal 2008 at orthocj.com on $9^{\text {th }}$ Oct, 2013

[8]. Weef, Jackson IT, Crookendale WA et.al. A case of thalassemia major with gross dental \& jaw deformities. Br. Journal of Maxillofacial Surgery1987;25:348-52

[9]. Cutando A. Thalassemias and their dental implications. Medicinia Oral2002;7:41-45

[10]. Tunaci M, Tunaci A, Engin G et.al. Imaging features of thalassemia. European Radiology 1999;9:1804-1809

[11]. Pope E, Berkovitch M, Klein J, et al. Salivary measurement of deferiprone concentrations and correlation with serum levels. Ther Drug Monit 1997; 19:95-7

[12]. De Mattia D, Pettini PL,Sabato V, et.al. Oromaxillofacial changes in thalassemia major. Minnerva Pediatr 1996;48:11-20. 
[13]. Hazza'a Am, Aljamal G. Radiographic feature of the jaws and teethin thalassemia major. Dentomaxillofacial Radiology,2006;35:283-288

[14]. Greenberg M, Glick M, Ship JA. Burket's Oral Medicine, $11^{\text {th }}$ edition 2008

[15]. Currarino G, Erlandson ME. Premature fusion of epiphysis in Cooley's anemia. Radiology 1964;83:656

[16]. Cohen AR. New advances in iron chelation therapy. Hematology Am Soc Hematol Educ Program.2006:42-47.

[17]. Berdoukas V, Farmaki K, Carson S, et.al. Treating thalassemia major-related iron overload: the roleof deferiprone. Journal of Blood Medicine2012;3:119-129

[18]. Breuer W, Shvartsman M, Cabantchik Zi. Intracellular labile iron. Int J Biochem Cell Biol. 2008;40(3):350-354

[19]. Borgna-Pignatti C, Rugolotto S, De Stefano P, et al. Survival and complications in patients with thalassemia major treated with transfusion and deferoxamine. Haematologica. 2004;89(10):1187-93

[20]. Wood JC. Diagnosis and management of transfusion iron overload: the role of imaging. Am J Hematol.2007;82(Suppl 12): 11321135

[21]. Angelucci E, Brittenham GM, McLaren CE, et al. Hepatic iron concentration and total body iron stores in thalassemia major. N Engl J Med 2000;343(5):327-331

[22]. Noetzli LJ, Carson SM, Nord AS, et al. Longitudinal analysis of heart and liver iron in thalassemia major. Blood 2008;112(7):29732978

[23]. Propper RD, Cooper B, Rufo RR, et al. Continuous subcutaneous administration of deferroxamine in patients with iron overload. N Engl J Med. 1977;297(8):418-423

[24]. Porter JB, Shah FT. Iron overload in thalassemia and related conditions: therapeutic goals and assessment of response to chelation therapies. Hematol Oncol Clin North Am.2010;24(6):1109-30

[25]. Pennell DJ, Berdoukas V, Karagiorga M, et al. Randomized controlled trial of deferiprone or deferoxamine in Beta- thalassemia major patients with asymptomatic myocardial siderosis. Blood. 2006;107(9):3738-44

[26]. Rachmilewitz EA, Aker M. the role of recombinant human erythropoietin in the treatment of thalassemia. Ann N Y Acad Sci 1998;850:129-38

[27]. Hajjar FM, Pearson HA. Pharmacologic treatment of thalassemia intermedia with hydroxyurea. J Pediatr 1994;125:490-492

[28]. Piomelli S. Management of Cooley's anaemia. Clin. Haematol. 1993;6:287-98

[29]. Borgna- Pignatti C, Vergine G, Lombardo T, et al. Hepatocellular carcinoma in the thalassemia syndromes. Br J Haematol. 2004;124(1);114-117 